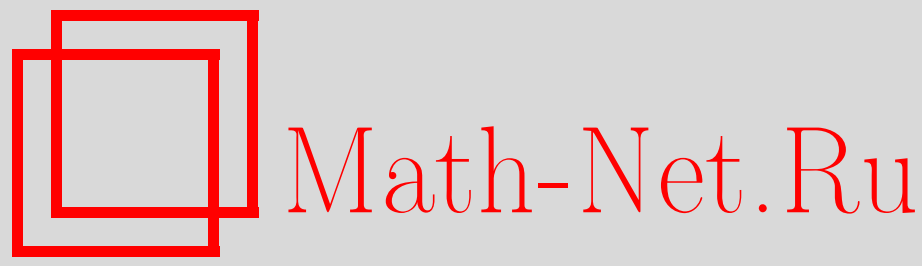

Н. Б. Ильин, А. Н. Печень, Критическая точка в задаче максимизации вероятности перехода с помощью измерений в $n$-уровневой квантовой системе, ТМФ, 2018, том 194, номер 3, 445-451

DOI: https://doi.org/10.4213/tmf9346

Использование Общероссийского математического портала Math-Net.Ru подразумевает, что вы прочитали и согласны с пользовательским соглашением http: //www . mathnet.ru/rus/agreement

Параметры загрузки:

IP : 54.197 .217 .227

26 апреля 2023 г., 15:53:28

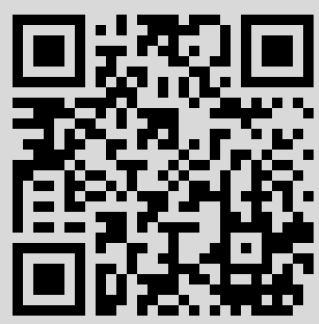




\title{
КРИТИЧЕСКАЯ ТОЧКА В ЗАДАЧЕ МАКСИМИЗАЦИИ ВЕРОЯТНОСТИ ПЕРЕХОДА С ПОМОЩЬЮ ИЗМЕРЕНИЙ В $n$-УРОВНЕВОЙ КВАНТОВОЙ СИСТЕМЕ
}

\begin{abstract}
Рассматривается задача максимизации вероятности перехода $n$-уровневой квантовой системы из заданного начального состояния в заданное конечное с помощью неселективных квантовых измерений. Найдена последовательность измерений, которая является критической точкой вероятности перехода и, кроме того, локальным максимумом по каждой из переменных на множестве одномерных проекторов. Класс одномерных проекторов рассматривается потому, что такие проекторы описывают измерения населенностей чистых состояний системы.
\end{abstract}

Ключевые слова: многоуровневая квантовая система, открытая квантовая система, квантовые измерения, управление квантовыми системами.

DOI: https://doi.org/10.4213/tmf9346

\section{1. ВВЕДЕНИЕ}

Управление квантовыми системами является активно развивающейся областью квантовой механики [1]-[5]. Манипулирование квантовыми системами находит применение в задачах квантовых технологий, управления химическими реакциями, для квантовых вычислений, оптимального разделения изотопов, создания квантовых корреляций [6]-[11] и других. Управление может осуществляться с помощью различных способов воздействия на систему. Одним из способов является когерентное управление, при котором варьируются параметры, определяющие гамильтониан системы. В этом случае изменяется потенциал взаимодействия системы с внешним управляющим полем, например лазерным импульсом, интенсивность которого модулируется для достижения цели управления.

Работа выполнена в рамках задания № 1.669.2016/ФПМ.

* Математический институт им. В. А. Стеклова Российской академии наук, Москва, Россия. E-mail: ilyn@mi.ras.ru

${ }^{\dagger}$ Национальный исследовательский технологический университет "МИСиС", Москва, Россия. E-mail: pechen@mi.ras.ru 
Другой способ - некогерентное управление с помощью резервуара или с помощью квантовых измерений. При некогерентном управлении с использованием измерений в определенные моменты времени над системой производятся неселективные измерения. Квантовые измерения широко используются для задач квантовой информации [12], [13]. Изучается управление квантовыми системами с вырожденными состояниями [14], динамика волнового пакета на торе [15]. В соответствии с постулатами квантовой механики измерение какой-либо величины оказывает влияние на состояние системы. Такое влияние может использоваться как управляющее воздействие [16]-[19]. Этот способ управления используется в квантовом эффекте Зенона и анти-Зенона [20], [21], когда с помощью специально подобранных управлений осуществляется управляемая эволюция квантовой системы из начального состояния в заданное целевое. Квантовые измерения применяются для управления кубитом и другими квантовыми системами [22], [23], для квантовых вычислений [24], управления вероятностью перехода в системе Ландау-Зинера [25]. Рассматриваются как дискретные, так и непрерывные измерения [26], [27]. Изучается влияние измерений на работу, производимую над квантовыми системами [28]. Применяется машинное обучение для реализации точных квантовых измерений [29].

Для оптимального управления квантовой системой необходимо найти глобальный максимум целевого функционала данной задачи [30]-[36]. В работе [16] найдены измерения, реализующие глобальный максимум в задаче оптимизации вероятности перехода из заданного начального состояния $\left|\psi_{\text {in }}\right\rangle$ в заданное целевое состояние $\left|\psi_{\text {fin }}\right\rangle$ двухуровневой квантовой системы. На основе этого результата построена последовательность измерений, являющаяся экстремалью относительно вариаций наблюдаемых, которые задаются проекторами на векторы в подпространстве $L$, натянутом на $\left|\psi_{\text {in }}\right\rangle$ и $\left|\psi_{\text {fin }}\right\rangle$. Возможно, что эта последовательность измерений в действительности доставляет глобальный максимум. Однако эта гипотеза на данный момент не доказана. В настоящей работе доказано более слабое утверждение о том, что эта последовательность измерений является критической точкой вероятности перехода на множестве наблюдаемых, являющихся одномерными проекторами. Более того, доказано, что она является точкой локального максимума по каждой из измеряемых наблюдаемых при фиксированных значениях остальных. Рассмотрение одномерных проекторов определяется тем, что они описывают практически наиболее важный класс измерений, соответствующий измерению населенностей чистых состояний квантовой системы.

\section{2. МАКСИМИЗАЦИЯ ВЕРОЯТНОСТИ ПЕРЕХОДА В $n$-УРОВНЕВОЙ СИСТЕМЕ С ПОМОЩЬЮ ИЗМЕРЕНИЙ}

Пусть в моменты времени $t_{k}$ в $n$-уровневой квантовой системе измеряются наблюдаемые $Q_{k}$. В результате неселективного измерения наблюдаемой $Q$ матрица плотности системы преобразуется как

$$
\rho \rightarrow \mathcal{M}_{Q}(\rho):=\sum_{i} P_{i} \rho P_{i}
$$

Здесь $P_{i}$ - проекторы на собственные подпространства наблюдаемой $Q$, которые определяются ее спектральным представлением $Q=\sum_{i} q_{i} P_{i}$. Между измерениями матрица плотности преобразуется с помощью оператора унитарной эволюции $U_{t}=e^{-i H t}$ :

$$
\rho \rightarrow \mathcal{U}_{t}(\rho):=e^{-i H t} \rho e^{i H t} .
$$

Здесь $H$ - гамильтониан системы (эрмитова $(n \times n)$-матрица). 
Результирующее преобразование матрицы плотности под воздействием измерений величин $Q_{k}$ в моменты времени $t_{k}$ и унитарной эволюции на отрезках $\left[t_{k}, t_{k+1}\right]$ имеет вид

$$
\rho_{T}=\mathcal{U}_{\left(T-t_{N}\right)} \circ \mathcal{M}_{Q_{N}} \circ \mathcal{U}_{\left(t_{N}-t_{N-1}\right)} \circ \cdots \circ \mathcal{M}_{Q_{1}} \circ \mathcal{U}_{t_{1}}\left(\rho_{0}\right)
$$

Здесь $T$ - конечный момент времени, $\rho_{0}$ - матрица плотности начального состояния системы.

Целевая функция для задачи максимизации вероятности перехода из начального состояния $\left|\psi_{\text {in }}\right\rangle$ в заданное конечное состояние $\left|\psi_{\text {fin }}\right\rangle \neq\left|\psi_{\text {in }}\right\rangle$ имеет вид

$$
\mathcal{P}_{N}\left(Q_{1}, \ldots, Q_{N}\right)=\left\langle\psi_{\text {fin }}\left|\mathcal{U}_{\left(T-t_{N}\right)} \circ \mathcal{M}_{Q_{N}} \circ \cdots \circ \mathcal{M}_{Q_{1}} \circ \mathcal{U}_{t_{1}}\left(\left|\psi_{\text {in }}\right\rangle\left\langle\psi_{\text {in }}\right|\right)\right| \psi_{\text {fin }}\right\rangle .
$$

Цель управления состоит в том, чтобы за время $T$ перевести систему с помощью $N$ неселективных измерений из начального состояния $\left|\psi_{\text {in }}\right\rangle$ как можно ближе к целевому состоянию $\left|\psi_{\text {fin }}\right\rangle$. Для этого необходимо найти оптимальные наблюдаемые $Q_{1}^{\mathrm{opt}}, \ldots, Q_{N}^{\mathrm{opt}}$, которые максимизируют вероятность перехода $\mathcal{P}_{N}\left(Q_{1}, \ldots, Q_{N}\right)$ :

$$
\mathcal{P}_{N}\left(Q_{1}^{\mathrm{opt}}, \ldots, Q_{N}^{\mathrm{opt}}\right)=\max _{Q_{1}, \ldots, Q_{N}} \mathcal{P}_{N}\left(Q_{1}, \ldots, Q_{N}\right) .
$$

Согласно статье [37] задача с собственной динамикой, задаваемой гамильтонианом $H$, эквивалентна задаче с тривиальной динамикой (т. е. при $H=0$ ) при замене $\left|\psi_{\text {in }}\right\rangle \rightarrow e^{-i T H}\left|\psi_{\text {in }}\right\rangle$. Поэтому в дальнейшем мы рассматриваем случай с тривиальной динамикой.

В работе [37] построены наблюдаемые $\widetilde{Q}_{k}$ такие, что

$$
\mathcal{P}_{N}\left(\widetilde{Q}_{1}, \ldots, \widetilde{Q}_{N}\right)=\frac{1}{2}\left(1+\left[\cos \frac{\Delta \varphi}{N+1}\right]^{N+1}\right),
$$

где

$$
\Delta \varphi=\arccos \left(2\left|\left\langle\psi_{\text {fin }} \mid \psi_{\text {in }}\right\rangle\right|^{2}-1\right) .
$$

Для построения этих наблюдаемых введем набор операторов $\boldsymbol{\sigma}=\left(\sigma_{x}, \sigma_{y}, \sigma_{z}\right)$, где $\sigma_{x}=\left|\psi_{\text {in }}\right\rangle\left\langle\psi_{\text {in }}^{\perp}|+| \psi_{\text {in }}^{\perp}\right\rangle\left\langle\psi_{\text {in }}\left|, \sigma_{y}=i\left(\left|\psi_{\text {in }}\right\rangle\left\langle\psi_{\text {in }}^{\perp}|-| \psi_{\text {in }}^{\perp}\right\rangle\left\langle\psi_{\text {in }}\right|\right), \sigma_{z}=\mathbb{I}-2\right| \psi_{\text {in }}\right\rangle\left\langle\psi_{\text {in }}\right|$. Здесь $\left|\psi_{\text {in }}^{\perp}\right\rangle=\psi /\|\psi\|$, где $\psi=\left|\psi_{\text {fin }}\right\rangle-\left\langle\psi_{\text {in }} \mid \psi_{\text {fin }}\right\rangle\left|\psi_{\text {in }}\right\rangle$ для невырожденного случая $\left|\psi_{\text {fin }}\right\rangle \neq$ $\left|\psi_{\text {in }}\right\rangle$. Определим также векторы $\mathbf{a}=\left\langle\psi_{\text {in }}|\boldsymbol{\sigma}| \psi_{\text {in }}\right\rangle$ и $\boldsymbol{\lambda}=\left\langle\psi_{\text {fin }}|\boldsymbol{\sigma}| \psi_{\text {fin }}\right\rangle$. Тогда

$$
\widetilde{Q}_{k}=\alpha P_{k}+\beta\left(\mathbb{I}-P_{k}\right), \quad \alpha, \beta \in \mathbb{R}, \quad \alpha \neq \beta,
$$

где

$$
P_{k}=\frac{1}{2}\left[\mathbb{I}+\left(\mathbf{a}_{k} \cdot \boldsymbol{\sigma}\right)\right]
$$

Здесь для $\Delta \varphi \in[0, \pi)$

$$
\mathbf{a}_{k}=\mathbf{a} \frac{\sin \left[\frac{N-k+1}{N+1} \Delta \varphi\right]}{\sin \Delta \varphi}+\lambda \frac{\sin \left[\frac{k}{N+1} \Delta \varphi\right]}{\sin \Delta \varphi}, \quad k=1, \ldots, N
$$

для $\Delta \varphi=\pi$

$$
\mathbf{a}_{k}=\mathbf{a} \operatorname{acos}\left[\frac{k}{N+1} \pi\right]+\tilde{\mathbf{a}} \sin \left[\frac{k}{N+1} \pi\right], \quad k=1, \ldots, N,
$$

где $\mathbf{a}$ - любой единичный вектор, ортогональный вектору а.

В статье [37] показано, что последовательность измерений $\widetilde{Q}_{1}, \ldots, \widetilde{Q}_{N}$ реализует максимум вероятности перехода на множестве наблюдаемых, заданных проекторами на векторы из подпространства $L$, натянутого на $\left|\psi_{\text {in }}\right\rangle$ и $\left|\psi_{\text {fin }}\right\rangle$. 


\section{3. КРИТИЧЕСКАЯ ТОЧКА ВЕРОЯТНОСТИ ПЕРЕХОДА В КЛАССЕ ОДНОМЕРНЫХ ПРОЕКТОРОВ}

Утверждение данной работы о том, что последовательность $\widetilde{Q}_{1}, \ldots, \widetilde{Q}_{N}$ является критической точкой на множестве последовательностей одномерных проекторов $\mathcal{Q}=\left\{\left(P_{1}, \ldots, P_{N}\right): P_{k} \in \mathbb{C}^{n \times n}, P_{k}=P_{k}^{\dagger}, P_{k}=P_{k}^{2}, \operatorname{rank} P_{k}=1, k=1, \ldots, N\right\}$, вытекает из следующей теоремы.

Теорема 1. В задаче максимизации вероятности перехода $\mathcal{P}_{N}\left(Q_{1}, \ldots, Q_{N}\right)$ на множестве $\mathcal{Q}$ для п-уровневой квантовой системы последовательность наблюдаемых $\widetilde{Q}_{1}, \ldots, \widetilde{Q}_{N}$, определенных выражением (8), является точкой локального максимума по каждой из переменных $Q_{i}$ при фиксированных значениях остальных.

ДокАЗАтЕЛьСтво. Целевая функция на множестве $\mathcal{Q}$ имеет вид

$$
\mathcal{P}_{N}\left(P_{1}, \ldots, P_{N}\right)=\operatorname{Tr}\left[\rho_{T} O\right],
$$

где $\rho_{T}=\mathcal{M}_{P_{N}} \circ \cdots \circ \mathcal{M}_{P_{1}}\left(\rho_{0}\right), P_{k}=\left|\psi_{k}\right\rangle\left\langle\psi_{k}\left|, \rho_{0}=\right| \psi_{\text {in }}\right\rangle\left\langle\psi_{\text {in }}\right|$ и $O=\left|\psi_{\text {fin }}\right\rangle\left\langle\psi_{\text {fin }}\right|$. В дальнейшем будем предполагать, что проектор $P_{k}, k=1, \ldots, N$, определяется формулой (9). Введем функцию от вектора $|\chi\rangle$

$$
\mathcal{P}_{N}^{\chi}:=\operatorname{Tr}\left[\mathcal{M}_{P_{N}} \circ \cdots \circ \mathcal{M}_{P_{\chi}} \circ \cdots \circ \mathcal{M}_{P_{1}}\left(\rho_{0}\right) O\right] .
$$

Используя равенство $\operatorname{Tr}\left[\mathcal{M}_{P}(\rho) O\right]=\operatorname{Tr}\left[\rho \mathcal{M}_{P}(O)\right]$, получаем, что

$$
\mathcal{P}_{N}^{\chi}=\operatorname{Tr}\left[\mathcal{M}_{P_{\chi}}\left(\rho_{k}\right) O_{k}\right],
$$

где $\rho_{k}=\mathcal{M}_{P_{k-1}} \circ \cdots \circ \mathcal{M}_{P_{1}}\left(\rho_{0}\right)$ и $O_{k}=\mathcal{M}_{P_{k+1}} \circ \cdots \circ \mathcal{M}_{P_{N}}(O)$. Имеем

$$
\mathcal{P}_{N}^{\chi}=\operatorname{Tr}\left[\rho_{k} O_{k}\right]-\left\langle\chi\left|\rho_{k} O_{k}+O_{k} \rho_{k}\right| \chi\right\rangle+2\left\langle\chi\left|\rho_{k}\right| \chi\right\rangle\left\langle\chi\left|O_{k}\right| \chi\right\rangle .
$$

Представим вектор $|\chi\rangle$ из некоторой окрестности вектора $\left|\psi_{k}\right\rangle$ на единичной сфере $S=\{|\chi\rangle:\|\chi\|=1\}$ с помощью пары векторов

$$
\left(\left|\chi^{\|}\right\rangle,\left|\chi^{\perp}\right\rangle\right) \in C, \quad C=\left\{\left(\left|\chi^{\|}\right\rangle,\left|\chi^{\perp}\right\rangle\right):\left|\chi^{\|}\right\rangle \in L,\left\|\chi^{\|}\right\|=1,\left|\chi^{\perp}\right\rangle \perp L,\left\|\chi^{\perp}\right\|<1\right\},
$$

по формуле $|\chi\rangle=\sqrt{1-\| \chi^{\perp}||^{2}}\left|\chi^{\|}\right\rangle+\left|\chi^{\perp}\right\rangle$. Формулы обратного преобразования имеют вид $\left|\chi^{\|}\right\rangle=\Pi_{L}|\chi\rangle / \| \Pi_{L}|\chi\rangle||,\left|\chi^{\perp}\right\rangle=|\chi\rangle-\Pi_{L}|\chi\rangle$, где $\Pi_{L}$ - проектор на подпространство $L$. При этом $\| \Pi_{L}|\chi\rangle \|=\sqrt{1-\left\|\chi^{\perp}\right\|^{2}}$. Отметим, что проектору $P_{\chi}=|\chi\rangle\langle\chi|$ соответствует класс эквивалентности векторов $|\chi\rangle \sim e^{i \alpha}|\chi\rangle, \alpha \in \mathbb{R}$, а в новых переменных - класс эквивалентности пар $\left(\left|\chi^{\|}\right\rangle,\left|\chi^{\perp}\right\rangle\right) \sim\left(e^{i \alpha}\left|\chi^{\|}\right\rangle, e^{i \alpha}\left|\chi^{\perp}\right\rangle\right)$. Поскольку все операторы $\rho_{0}, O, P_{1}, \ldots, P_{N}$ коммутируют с проектором $\Pi_{L}$, то $\rho_{k}=\Pi_{L} \rho_{k} \Pi_{L}$ и $O_{k}=\Pi_{L} O_{k} \Pi_{L}$. Тогда выражение (15) преобразуется в равенство

$\mathcal{P}_{N}^{\chi}=\operatorname{Tr}\left[\rho_{k} O_{k}\right]-\left(1-\left\|\chi^{\perp}\right\|^{2}\right)\left\langle\chi^{\|}\left|\rho_{k} O_{k}+O_{k} \rho_{k}\right| \chi^{\|}\right\rangle+2\left(1-\left\|\chi^{\perp}\right\|^{2}\right)^{2}\left\langle\chi^{\|}\left|\rho_{k}\right| \chi^{\|}\right\rangle\left\langle\chi^{\|}\left|O_{k}\right| \chi^{\|}\right\rangle$.

Рассмотрим разность $\delta \mathcal{P}_{N}=\mathcal{P}_{N}-\mathcal{P}_{N}^{\chi}$. Учитывая, что $\mathcal{P}_{N}=\mathcal{P}_{N}^{\psi_{k}}$, запишем ее в виде

$$
\delta \mathcal{P}_{N}=\mathcal{P}_{N}^{\psi_{k}}-\mathcal{P}_{N}^{\chi \|}+\mathcal{P}_{N}^{\chi \|}-\mathcal{P}_{N}^{\chi} .
$$

Используя формулу (16) при $|\chi\rangle=\left|\chi^{\|}\right\rangle$, получим

$$
\mathcal{P}_{N}^{\chi^{\|}}-\mathcal{P}_{N}^{\chi}=\left\|\chi^{\perp}\right\|^{2}\left(-\left\langle\chi^{\|}\left|\rho_{k} O_{k}+O_{k} \rho_{k}\right| \chi^{\|}\right\rangle+2\left(2-\left\|\chi^{\perp}\right\|^{2}\right)\left\langle\chi^{\|}\left|\rho_{k}\right| \chi^{\|}\right\rangle\left\langle\chi^{\|}\left|O_{k}\right| \chi^{\|}\right\rangle\right) .
$$


Обозначим через $\mathcal{P}_{N, k}=\operatorname{Tr}\left[\rho_{k} O_{k}\right]$ вероятность перехода для $N-1$ измерений наблюдаемых $\widetilde{Q}_{1}, \ldots, \widetilde{Q}_{k-1}, \widetilde{Q}_{k+1}, \ldots, \widetilde{Q}_{N}$. Разность $\mathcal{P}_{N}^{\chi \|}-\mathcal{P}_{N}^{\chi}$ имеет вид

$$
\mathcal{P}_{N}^{\chi^{\|}}-\mathcal{P}_{N, k}=-\left\langle\chi^{\|}\left|\rho_{k} O_{k}+O_{k} \rho_{k}\right| \chi^{\|}\right\rangle+2\left\langle\chi^{\|}\left|\rho_{k}\right| \chi^{\|}\right\rangle\left\langle\chi^{\|}\left|O_{k}\right| \chi^{\|}\right\rangle .
$$

Тогда соотношение (18) преобразуется в соотношение

$$
\mathcal{P}_{N}^{\chi^{\|}}-\mathcal{P}_{N}^{\chi}=\left\|\chi^{\perp}\right\|^{2}\left(\mathcal{P}_{N}^{\chi_{N}^{\|}}-\mathcal{P}_{N, k}+2\left(1-\left\|\chi^{\perp}\right\|^{2}\right)\left\langle\chi^{\|}\left|\rho_{k}\right| \chi^{\|}\right\rangle\left\langle\chi^{\|}\left|O_{k}\right| \chi^{\|}\right\rangle\right)
$$

Разность $\mathcal{P}_{N}^{\chi \|}-\mathcal{P}_{N, k}$ как функция вектора $\chi$ непрерывна, и ее значение в точке $\psi_{k}$ положительно. Действительно, согласно работе [37] для $\mathcal{P}_{N, k}$ имеем выражение

$$
\mathcal{P}_{N, k}=\frac{1}{2}\left(1+\left(\boldsymbol{\lambda} \cdot \mathbf{a}_{N}\right) \ldots\left(\mathbf{a}_{k+1} \cdot \mathbf{a}_{k-1}\right) \ldots\left(\mathbf{a}_{2} \cdot \mathbf{a}_{1}\right)\left(\mathbf{a}_{1} \cdot \mathbf{a}\right)\right) .
$$

При этом $\left(\mathbf{a}_{1} \cdot \mathbf{a}\right)=\left(\mathbf{a}_{2} \cdot \mathbf{a}_{1}\right)=\cdots=\left(\boldsymbol{\lambda} \cdot \mathbf{a}_{N}\right)=\cos [\Delta \varphi /(N+1)]$ и $\left(\mathbf{a}_{k+1} \cdot \mathbf{a}_{k-1}\right)=$ $\cos [2 \Delta \varphi /(N+1)]$. Отсюда для $\mathcal{P}_{N, k}$ получаем следующую формулу:

$$
\mathcal{P}_{N, k}=\frac{1}{2}\left(1+\cos \frac{2 \Delta \varphi}{N+1}\left[\cos \frac{\Delta \varphi}{N+1}\right]^{N-1}\right) .
$$

Здесь и далее неопределенность, возникающая в выражении $\cos ^{N-1}[\Delta \varphi /(N+1)]$ при $N=1$ и $\Delta \varphi=\pi$, доопределяется значением единица по непрерывности рассматриваемых величин по $\Delta \varphi$. Аналогично, для $\mathcal{P}_{N}^{\psi_{k}}$ имеет место формула

$$
\mathcal{P}_{N}^{\psi_{k}}=\frac{1}{2}\left(1+\left[\cos \frac{\Delta \varphi}{N+1}\right]^{N+1}\right) .
$$

Таким образом, для разности $\mathcal{P}_{N}^{\psi_{k}}-\mathcal{P}_{N, k}$ получаем выражение

$$
\mathcal{P}_{N}^{\psi_{k}}-\mathcal{P}_{N, k}=\frac{1}{2}\left[\sin \frac{\Delta \varphi}{N+1}\right]^{2}\left[\cos \frac{\Delta \varphi}{N+1}\right]^{N-1} .
$$

Следовательно, существует такая $\varepsilon$-окрестность вектора $\left|\psi_{k}\right\rangle$, что для всех векторов $|\chi\rangle$ из этой окрестности выполняется строгое неравенство $\mathcal{P}_{N}^{\chi \|}-\mathcal{P}_{N, k}>0$. Отметим, что $\Delta \varphi \neq 0$ для рассматриваемого невырожденного случая $\left|\psi_{\text {in }}\right\rangle \neq\left|\psi_{\text {fin }}\right\rangle$. Поскольку $\rho_{k}$ и $O_{k}$ - положительные операторы, то также выполняется неравенство $\left\langle\chi^{\|}\left|\rho_{k}\right| \chi^{\|}\right\rangle\left\langle\chi^{\|}\left|O_{k}\right| \chi^{\|}\right\rangle \geqslant 0$. Таким образом, если $\left\|\chi^{\perp}\right\| \neq 0$, то равенство (20) влечет неравенство $\mathcal{P}_{N}^{\chi \|}-\mathcal{P}_{N}^{\chi}>0$ и, следовательно, равенство (17) влечет неравенство $\delta \mathcal{P}_{N}>0$. Если $\left\|\chi^{\perp}\right\|=0$, то $|\chi\rangle \in L$. Тогда задача сводится к двумерному случаю, для которого $\mathcal{P}_{N}^{\psi_{k}}-\mathcal{P}_{N}^{\chi}>0$ при $\left|\psi_{k}\right\rangle \neq e^{i \alpha}|\chi\rangle$ и, следовательно, также $\delta \mathcal{P}_{N}>0$. Теорема доказана.

\section{4. ЗАКЛЮЧЕНИЕ}

Рассмотрена задача максимизации вероятности перехода $\mathcal{P}_{N}\left(Q_{1}, \ldots, Q_{N}\right)$ из заданного начального состояния $\left|\psi_{\text {in }}\right\rangle$ в заданное целевое состояние $\left|\psi_{\text {fin }}\right\rangle$ под воздействием $N$ неселективных измерений в $n$-уровневой квантовой системе. Для любой размерности системы $n$ и любого фиксированного числа измерений $N$ найдена последовательность измерений, которая является критической точкой вероятности перехода на множестве одномерных проекторов. Кроме того, доказано, что при измерении этих наблюдаемых достигается локальный максимум по каждой из переменных $Q_{i}$ при фиксированных значениях остальных. 


\section{Список литературы}

[1] S. A. Rice, M. Zhao, Optical Control of Molecular Dynamics, Wiley, New York, 2000.

[2] M. Shapiro, P.W. Brumer, Principles of the Quantum Control of Molecular Processes, Wiley, Hobooken, 2003.

[3] D. J. Tannor, Introduction to Quantum Mechanics: A Time Dependent Perspective, Univ. Sci. Books, Sausalito, CA, 2007.

[4] D. D'Alessandro, Introduction to Quantum Control and Dynamics, Chapman \& Hall, Boca Raton, FL, 2008.

[5] V.S. Letokhov, Laser Control of Atoms and Molecules, Oxford Univ. Press, New York, 2007.

[6] C. Brif, R. Chakrabarti, H. Rabitz, "Control of quantum phenomena", Adv. Chem. Phys., 148 (2012), 1-76.

[7] S. J. Glaser, U. Boscain, T. Calarco, C. P. Koch, W. Köckenberger, R. Kosloff, I. Kuprov, B. Luy, S. Schirmer, T. Schulte-Herbrüggen, D. Sugny, F. K. Wilhelm, "Training Schrödinger's cat: quantum optimal control", Eur. Phys. J. D, 69 (2015), 279, 24 pp.

[8] L. Accardi, S. V. Kozyrev, A. N. Pechen, "Coherent quantum control of $\Lambda$-atoms through the stochastic limit", Quantum Information and Computing, QP-PQ: Quantum Probability and White Noise Analysis, 19, eds. L. Accardi, M. Ohya, N. Watanabe, World Sci., Singapore, 2006, 1-17.

[9] А.И. Зенчук, С. И. Доронин, "Удаленный контроль квантовыми корреляциями двухкубитного приемника посредством трехкубитного передатчика", ТМФ, 188:2 (2016), 343-358.

[10] K. A. Lyakhov, H. J. Lee, A. N. Pechen, "Some features of Boron isotopes separation by the laser-assisted retardation of condensation method in multipass irradiation cell implemented as a resonator", IEEE J. Quantum Electron., 52:12 (2016), 1400208, 8 pp.

[11] S. V. Kozyrev, A. A. Mironov, A.E. Teretenkov, I. V. Volovich, "Flows in nonequilibrium quantum systems and quantum photosynthesis", Infin. Dimens. Anal. Quantum Probab. Relat. Top., 20:4 (2017), 1750021.

[12] A. S. Holevo, Statistical Structure of Quantum Theory, Lecture Notes in Physics. Monographs, 67, Springer, Berlin, 2001.

[13] M. Ohya, I. Volovich, Mathematical Foundations of Quantum Information and Computation and Its Applications to Nano- and Bio-Systems, Springer, Dordrecht, 2011.

[14] И. В. Волович, С. В. Козырев, "Манипуляция состояниями вырожденной квантовой системы", Современные проблемы математики, механики и математической физижи. IІ, Тр. МИАН, 294, 2016, 256-267.

[15] A. Trushechkin, "Semiclassical evolution of quantum wave packets on the torus beyond the Ehrenfest time in terms of Husimi distributions", J. Math. Phys., 58:6 (2017), 062102, 16 pp., arXiv: 1607.07572.

[16] A. N. Pechen, N. B. Ilin, F. Shuang, H. Rabitz, "Quantum control by von Neumann measurements", Phys. Rev. A, 74:5 (2006), 052102, 7 pp.

[17] H. W. Wiseman, "Quantum control: squinting at quantum systems", Nature, 470 (2011), 178-179.

[18] J. Gough, V.P. Belavkin, O. G. Smolyanov, "Hamilton-Jacobi-Bellman equations for quantum optimal feedback control", J. Opt. B: Quantum Semiclass. Opt., 7:10 (2005), S237-S244.

[19] R. Vilela Mendes, V. I. Man'ko, "Quantum control and the Strocchi map", Phys. Rev. A, 67:5 (2003), 053404, 8 pp.

[20] B. Misra, E. C. G. Sudarshan, "The Zeno's paradox in quantum theory", J. Math. Phys., 18:4 (1977), 756-763.

[21] A. P. Balachandran, S. M. Roy, "Quantum anti-Zeno paradox", Phys. Rev. Lett., 84:18 (2000), 4019-4022. 
[22] F. Shuang, A. N. Pechen, T.S. Ho, H. Rabitz, "Observation-assisted optimal control of quantum dynamics", J. Chem. Phys., 126:13 (2007), 134303, 7 pp.

[23] M. S. Blok, C. Bonato, M. L. Markham, D. J. Twitchen, V. V. Dobrovitski, R. Hanson, "Manipulating a qubit through the backaction of sequential partial measurements and real-time feedback", Nature Phys., 10 (2014), 189-193.

[24] G. A. Paz-Silva, A.T. Rezakhani, J. M. Dominy, D. A. Lidar, "Zeno effect for quantum computation and control", Phys. Rev. Lett., 108:8 (2012), 080501, 5 pp.

[25] A. N. Pechen, A. S. Trushechkin, "Measurement-assisted Landau-Zener transitions", Phys. Rev. A, 91:5 (2015), 052316, 15 pp.

[26] М. Г. Иванов, "О единственности квантовой теории измерений для точных измерений с дискретным спектром", Тр. МФТИ, 8:1(29) (2016), 170-178.

[27] М. Б. Менский, “Эволюция квантовой системы, подвергающейся непрерывному измерению", ТМФ, 75:1 (1988), 41-52.

[28] M. Campisi, P. Talkner, P. Hanngi, "Influence of measurements on the statistics of work performed on a quantum system", Phys. Rev. E, 83:4 (2011), 041114, 7 pp.

[29] A. Hentschel, B. C. Sanders, "Machine learning for precise quantum measurement", Phys. Rev. Lett., 104:6 (2010), 063603, 4 pp.

[30] H. Rabitz, H. Hsieh, C. Rosenthal, "Quantum optimally controlled transition landscapes", Science, 303:5666 (2004), 1998-2001.

[31] T.S. Ho, H. Rabitz, "Why do effective quantum controls appear easy to find?", J. Photochem. Photobiol. A, 180:3 (2006), 226-240.

[32] P. de Fouquieres, S. G. Schirmer, "A closer look at quantum control landscapes and their implication for control optimization", Infin. Dimens. Anal. Quantum Probab. Relat. Top., 16:3 (2013), 1350021, 24 pp.

[33] N. Rach, M. M. Müller, T. Calarco, S. Montangero, "Dressing the chopped-random-basis optimization: a bandwidth-limited access to the trap-free landscape", Phys. Rev. A, 92:6 (2015), 062343, 7 pp.

[34] А.Н. Печень, Н. Б. Ильин, "О критических точках целевого функционала в задаче максимизации наблюдаемых кубита", УМН, 70:4(424) (2015), 211-212.

[35] А.Н. Печень, "О методе скоростного градиента для генерации унитарных квантовых операций в замкнутых квантовых системах", УМН, 71:3(429) (2016), 205-206.

[36] A. N. Pechen, N.B. Il'in, "Control landscape for ultrafast manipulation by a qubit", J. Phys. A: Math. Theor., 50:7 (2017), 075301, 13 pp.

[37] А. Н. Печень, Н. Б. Ильин, "О задаче максимизации вероятности перехода в $n$-уровневой квантовой системе с помощью неселективных измерений”, Тр. МИАН, 294 (2016), 248-255.

Поступила в редакцию 31.01.2017, после доработки 9.08.2017 